\title{
Detection of Behavioral Patterns for Increasing Attentiveness Level
}

\author{
Dalila Durães ${ }^{1}$, Sérgio Gonçalves ${ }^{2}$, Davide Carneiro ${ }^{3,4}$, Javier Bajo ${ }^{1}$, Paulo Novais ${ }^{3}$ \\ ${ }^{1}$ Department of Artificial Intelligence, Technical University of Madrid, Madrid, Spain \\ d.alves@alumnos.upm.es, jbajo@fi.upm.es \\ ${ }^{2}$ Informatics Department, University of Vigo, Ourense, Spain \\ sgoncalvesealumnos.uvigo.es \\ ${ }^{3}$ Algoritmi Center, Minho University, Braga, Portugal \\ dcarneiroedi.uminho.pt, pjonedi.uminho.pt \\ ${ }^{4}$ CIICESI, ESTGF, Polytechnic Institute of Porto, Portugal
}

\begin{abstract}
In the current world, performance is one of the most important issues concerning work and competition. Performance is strongly connected with learning and when it comes to acquiring new knowledge, attention is one the most important mechanisms as the level of the learner's attention affects learning results. When students are doing learning activities using new technologies, it is extremely important that the teacher has some feedback from the students' work in order to detect potential learning problems at an early stage. The goal of this research is to propose a system that measures the level of attentiveness in real scenarios, and detects patterns of behavior associated to different attention levels among different students. This system measures attention and uses this information for training a decision support system that shows the level of attention of a group of students in real time.
\end{abstract}

Keywords: Ambient Intelligence, Learning Activities, Innovative Scenarios, and Attentiveness.

\section{$1 \quad$ Introduction}

Education, training, skills development, and learning are processes that are continuously performed since we are born. These characteristics and the ability to learn and teach, allow us to grow as a person. Besides, learning is inevitably linked to the History of Humanity, to its construction as a social being capable of adapting to new situations. The learning process is described as the act of acquiring knowledge, behaviors, abilities, standards or preferences and the study of learning has been closely linked to the development of psychology as a science [1].

Many contemporary educators argue the value of a constructivist approach to teaching. Advocates argue that constructivist learning better equips learners to successfully master new and novel situations [2]. It emphasizes problem solving over the mere accumulation of facts. One of the central arguments for the use of Web-based resources in the classroom is that it gives learners access to information resources in

adfa, p. 1, 2011.

(C) Springer-Verlag Berlin Heidelberg 2011 
ways that allow them to search for relevant data, synthesize that information, and draw their own conclusions.

For these reasons, the school must create systems that are capable of involving the students and capture their attention. Advances in computers and wireless technologies have also had an impact on the educational setting, thus generating a new approach for Technology-Enhanced Learning (TEL). The rapid development of these technologies combined with access to content in a wide variety of settings, allows learners to experience new learning situations beyond the school's walls [3].

The concept of adaptively refers to the idea that different learning contexts should be adaptable to the particular setting in which the learner is situated [3].

Being a cognitive process, attention is strongly connected with learning [4]. When it comes to acquiring new knowledge, attention can be considered one of the most important mechanisms [5]. The level of the learner's attention affects learning results. The lack of attention can define the success of a student. In learning activities, attention is also very important to perform these tasks in an efficient and adequate way.

There is no universally accepted definition of attention because there is a diversity of disciplines that study it. In the past, only psychologists studied attention; however attention is currently highly important for fields such as philosophy, chemistry, anatomy, and even computational science [6]. With the existence of these multiple sets of disciplines that study attention, its definition diverges depending on the field of study. For example, we can differentiate the concept of attention in human beings and machines. In humans, attention is processed in the brain while in machines there is a processor unit with a certain memory capacity that will process data. As with the brain, computers should analyze more and more data, but unlike the brain they do not or do rarely, "pay attention" [7] to the data.

Attention is the cognitive process of focusing on one aspect of the environment while disregarding others. "Pay attention!" is a phrase repeated by so many teachers all over world, and the explanation for this is simple [7]. Attention is the first step in the learning process. Students cannot learn or understand or even remember if they do not listen properly, so, they will fail in the learning process. For almost everyone it is easy to pay attention to subjects or things that are interesting or stimulating to them.

So the target of this paper is to propose the architecture of an ambient intelligent system aimed at capturing and measuring the level of the students' attentiveness in real scenarios and dynamically provide recommendations to the teacher. The students can learn in a relatively anonymous environment without the embarrassment of failure and/or socio-cultural prejudice from personal contact. All the students get the same standardized set of learning materials from the learning activities using technologies in an on-line environment. This environment is collaborative. The use of groups and teams working together in collaborative learning contexts allow interactions which enforce employability skills. Teaching other subjects they have learned help them to reinforce the learning process. The student usage of the learning materials can be monitored and early possible dropout can be discovered and counteractive support can be offered. The on-line environments can be used to give instant feedback from online self-assessment or formative assessment particularly through multiple choice question formats. In addition, the students can be offered automatically marked self-assessment 
exercises to ascertain skill/knowledge levels and learning needs before engaging with course content. It can also assist students with certain disabilities.

\section{$2 \quad$ Proposed Design}

When students are affected by positive or negative states, they produce different kinds of thinking and this might hold important implications on the educational and training perspective. When that occurs it would be important to be able to notify and advise the teacher, so that she/he can be able to dynamically modify the teaching style according to students' feedback signals which include cognitive, emotional and motivational aspects.

While the student conscientiously interacts with the system and takes his/her decisions and actions, a parallel and transparent process takes place in which the TEL system uses the information. The architecture of the TEL system presented in Figure 1 depicts the process through which the system operates.

The devices in which students work have software that generates raw data. These devices store the raw data locally until it is synchronized with the web server in the cloud, which occurs at regular intervals (normally 5 minutes).

In the storage layer the raw data received from the data generating devices is stored in a data store engine. The analytic layer provides powerful tools for performing analytics and analyses in real-time. The system calculates, at regular intervals, an estimation of the general level of performance and attention of each student.

The classification layer is where the indicators are interpreted. For example, interpreting data from the attentiveness indicators and building the meta-data that will support decision-making. When the system has a sufficiently large dataset that allows making classifications with precision, it will classify the inputs received into different attention levels in real-time. This layer has access to the current and historical state of the group from a global perspective, but it can also refer to each student individually creating each student learning profile.

With the results concerning the level of attention and the alternative strategies applied during a long period of time by the teacher, it is possible to obtain a profile of the learning style. This meta-data will allow the system to create the indicators used to perform decision-making.

The classification is also a very important aspect to have control of. This possibility allows analyses within longer time frames. This information will be used by another sub-module, the affective adaptive agent, to provide relevant information to the platform and to the mentioned personalization module.

Finally, the Client layer is developed as a web app with an intuitive and visual representations (diagrams and graphical) of the attentiveness state of the group and of each student, abstracting from the complexity of the data level where they are positioned. At this point, the system can start to be used by the people involved, especially the teacher who can better adapt and personalize her/his teaching strategies. With a focus on individual and group performance and using real time analytics, the visual representation tools are intuitive, suggesting and facilitating the decision-making and 
human resources management. The actual students' attention information is displayed in the visualization layer, and can be used to personalize instruction according to the specific student, enabling the teacher to act differently with different students, and also to act differently with the same student, according to his/her past and present level of attention.

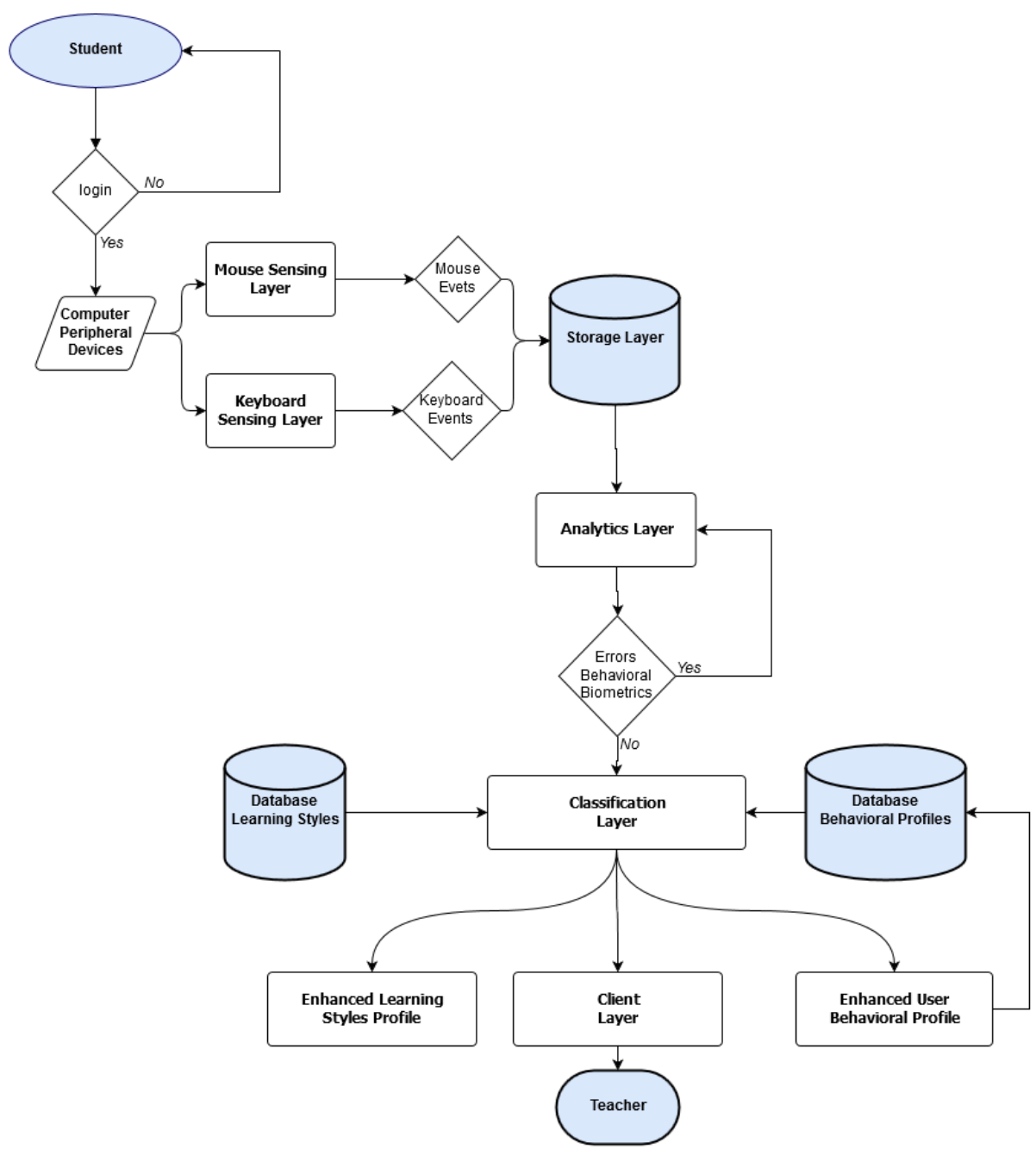

Fig. 1. Diagram of the system.

\subsection{Methodology}

In this work we compare two classes from the electronics vocational courses while performing an activity based on serious games at the High School of Caldas das Taipas, Guimarães, Portugal. We want to determine if classes from different level have a 
significant effect on mouse and keyboard dynamics, and attention level. With these results the TEL system can support future decision-making.

For this purpose, two groups of 10 (12I, all male students) and 7 (11J, all male students) electronic vocational students were selected to participate, whose average age is seventeen (12I) and sixteen (11J) years old. In the same week, they have a serious game lesson, where they have access to an individual computer and nineteen minutes to complete a task. For the 12I class, the lesson started at 8:30 and finished at 10:00 a.m, and for the 11J class, the lesson started at 11:30 a.m. and finished at 01:00 p.m. Students received, at the beginning of the lesson, a document with the goals of the task.

The first stage of the proposed system is the collection of the relevant data, which was designed and carried out using a logger application developed in previous work [8]. The data collected by the logger application characterizing the students' interaction patterns is aggregated in a server to which the logger application connects after the student logs in. The privacy of the students is ensured, since the necessary data that is collected to perform the login and the registration are an ID that does not personally identify the student, password, and the gender.

This application runs in the background, which makes the data acquisition process, a completely transparent one from the point of view of the student. The system was developed to acquire data from normal working routines, compiling information from the students' activities through the mouse and the keyboard, which act as sensors. The Mouse and Keyboard Sensing layers are responsible for capturing information describing the behavioral patterns of the students, and receiving data from the events generated by their mouse and keyboard [8]. These data are further processed, stored and then used to calculate the values of the behavioral biometrics.

\subsection{Data Analysis}

In this sub-section we will show the existence of different behaviors in the two different classes. Data was analyzed in two different ways. First, a general analysis was carried out in which statics methods are used to obtain preliminary conclusions. Second, an individual analysis was made in order to compare the different moments.

In a preliminary analysis of data, it was concluded that there are indeed different interaction patterns depending on the group analyzed. To conclude this, we looked at the distributions of the data collected and analyzed the statistical significance of their differences. To this end, we used the Kruskal-Wallis test. Table 1 details the mean value of each feature in each class (evaluation and normal). It also details the $p$-value of the Kruskal-Wallis test.

When data from the two classes is compared, the first conclusion is that the differences observed are statistically significant in nearly all features. Moreover, mean values of the features are consistently lower in the 12I class. In most of the features, this indicates an increased performance (e.g. a smaller average distance between clicks means that the student moved the mouse in a more efficient manner). In the case of mouse velocity, for example, a smaller velocity could point out a slower, and thus less efficient, movement. 
Table 1. Results of the Kruskall-Wallis test and mean values for each class and each feature.

\begin{tabular}{|c|c|c|c|}
\hline Feature & Significance & 121 & $11 \mathrm{~J}$ \\
\hline$k d t$ & 0.17397 & 158.60 & 348.28 \\
\hline$t b k$ & 0.00013 & 867.95 & 483.58 \\
\hline$m v$ & $5.63 \times 10^{-10}$ & 0,49 & 0,59 \\
\hline$m a$ & $6.40 \times 10^{-8}$ & 0,53 & 0,61 \\
\hline$c d$ & $6.10 \times 10^{-21}$ & 93,95 & 509,40 \\
\hline$t b c$ & $3.39 \times 10^{-10}$ & 3981,61 & 2352,06 \\
\hline$d b c$ & 0.569996 & 213,39 & 206,13 \\
\hline$d d c$ & $4.93 \times 10^{-21}$ & 81,93 & 272,36 \\
\hline$e d b c$ & 0.004164 & 395,33 & 306,82 \\
\hline$a e d b c$ & $5.53 \times 10^{-11}$ & 2,84 & 1,89 \\
\hline$s s d b c$ & 0.0037082 & $-0,46$ & $-10,03$ \\
\hline$a s d b c$ & $1.41 \times 10^{-6}$ & 6230,54 & 4557,29 \\
\hline$t d c$ & 0.632319 & 57,73 & 49,08 \\
\hline$d p / b c$ & $2.41 \times 10^{-6}$ & $1,543 \times 10^{7}$ & $2,132 \times 10^{7}$ \\
\hline adplbc & $2.09 \times 10^{-13}$ & 83112,50 & 170520,00 \\
\hline
\end{tabular}

In past work we concluded that a slower mouse velocity is indeed necessary for the student to achieve increased accuracy in mouse movement: moving the mouse too fast would make precise movements more difficult to carry out. A similar trend happens with mouse acceleration and click duration. The remaining features consistently show increased performance in the 12I class. Also for the keyboard features, the data are consistent with the features extracted from the mouse.

We analyzed all the applications used by all students and labelled each one of them as belonging to the task or not. We then quantified the amount of time that each student spent interacting with applications related to the task versus other applications.

For that, it is necessary to compute the level of attention of the user as detailed in Algorithm 1.

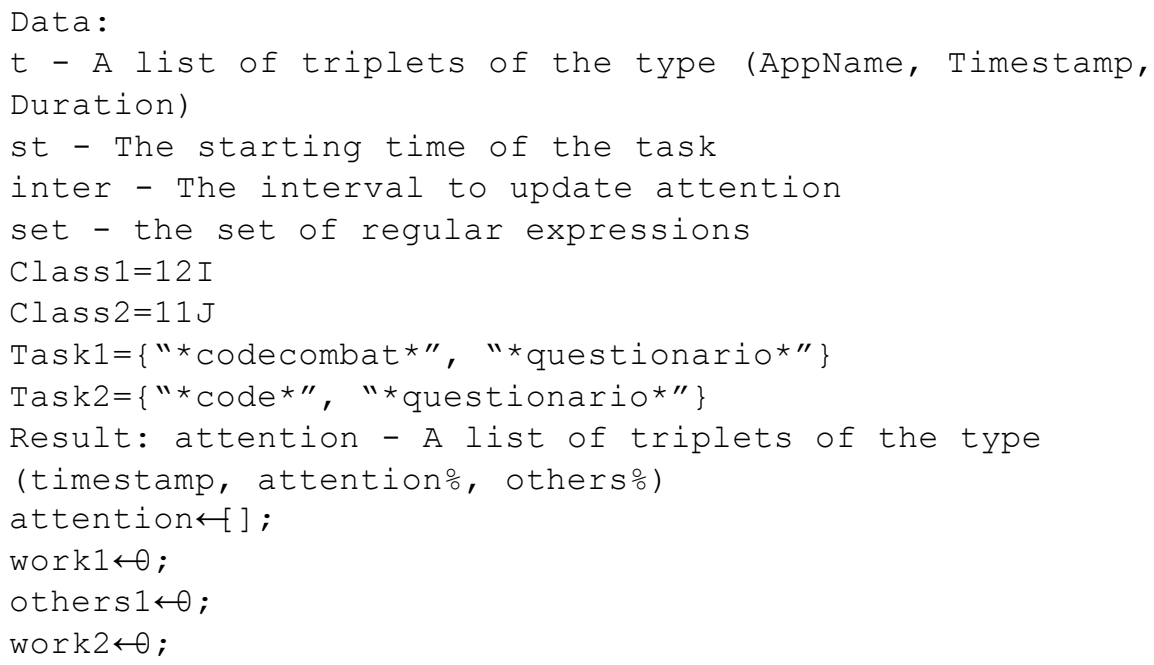




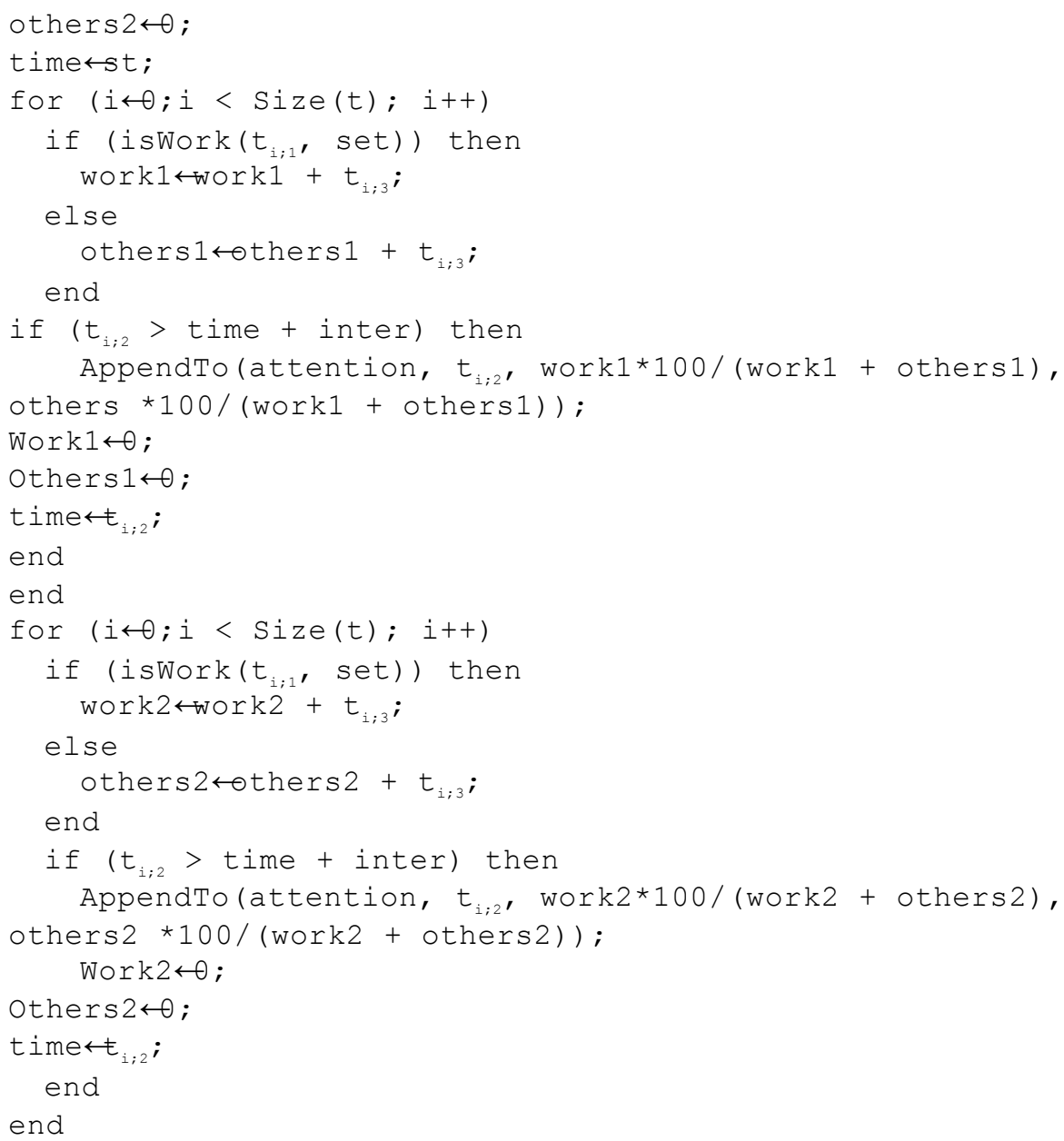

Algorithm 1: Creating triplets at regular intervals with the timestamp the quantification of attention.

To do this we measure the amount of time, in each interval, that the user spent interacting with work-related applications. The algorithm thus needs knowledge about the domain in order to classify each application as belonging or not to the set of workrelated applications. This knowledge is provided by the teacher and is encoded in the form of regular expressions. The teacher uses a graphical interface to set up rules such as "started with "codecombat" or "contains "code", which are then translated to regular expressions that are used by the algorithm to determine which applications are work-related and which are not.

Whenever an application that does not match any of the known rules for the specific domain is found, the application name is saved so that the teacher can later decide if a new rule should or should not be created for it. By default, applications that are 
not considered work-related are marked as "others" and count negatively towards the quantification of attention.

\section{$3 \quad$ Preliminary Results}

During the lessons with the two classes, the monitoring system was used to assess the interaction of the students with the computer and to quantify their level of attentiveness as well. To quantify attentiveness the following methodology was followed. Asides from capturing the interaction of the students with the computer, the monitoring system also registers the applications with which students are interacting.

Attention is calculated at regular intervals, as configured by the teacher (e.g. five minutes). The teacher may also want to assess, in real-time or a posteriori, the evolution of attention of the whole class. Figure 2 presents the evolution of attention for each class in a lesson. If necessary, the teacher may also click on a student to analyze the temporal evolution of the attention for that specific student during the class.
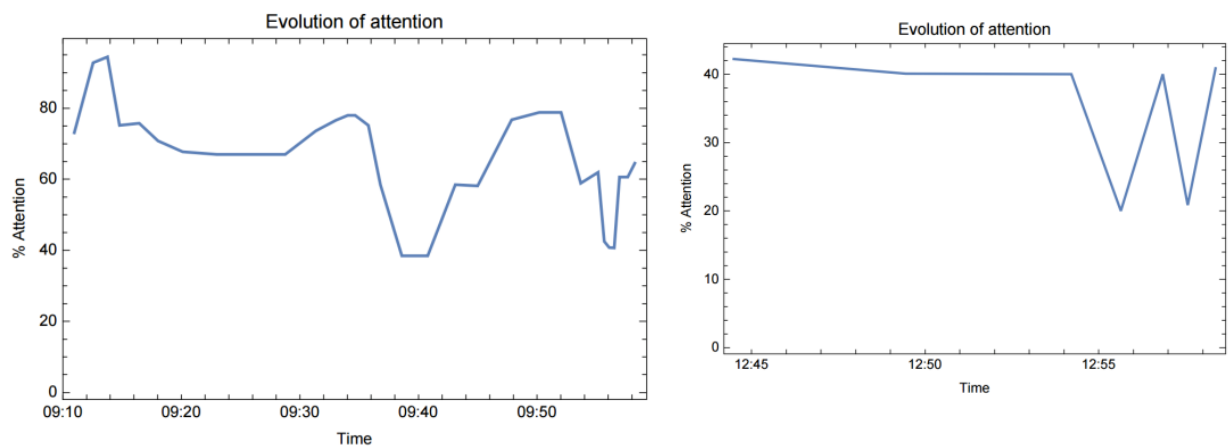

Fig. 2. Temporal evolution of attention in the two class (a) 12I (b) $11 \mathrm{~J}$.

Table 2. Percentage of work and the scores obtained by the students: left - 12I; right - 11J

\begin{tabular}{||c|c|c||c||c||c||}
\hline Student & \% Attention & Results & Student & \% Attention & Results \\
\hline T2210001 & 78,2417 & 14 & T7110061 & 0.0648998 & 6 \\
\hline T2210002 & 62.6977 & 12 & T7110062 & 48,093 & 6 \\
\hline T2210003 & 52.1404 & 12 & T7110064 & 95.9514 & 14 \\
\hline T2210004 & 82.0908 & 15 & T7110066 & 84.2233 & 13 \\
\hline T2210006 & 49,5761 & 8 & T7110068 & 4,21712 & 8 \\
\hline T2210007 & 44.2481 & 8 & T7110072 & 100 & 14 \\
\hline T2210008 & 86.6918 & 13 & T7110073 & 6.19405 & 6 \\
\hline T2210009 & 98.7981 & 16 & & & \\
\hline T2210010 & 94.3469 & 17 & & & \\
\hline T2210012 & 55,3309 & 13 & & & \\
\hline
\end{tabular}

The correlation between the level of attentiveness and the score of the students in the task in each class was also analyzed. In the two classes, there are positive correlations: 0.875 in $12 \mathrm{I}$ and 0.90 in $11 \mathrm{~J}$. We believe that these values are so high for two reasons: (1) these classes are mostly composed of two very different types of students 
(bad and excellent students) and (2) the task was of high difficulty. Finally, there were many differences in the scores. In future work we will study this relationship in more detail, namely in different classes and with tasks of different levels of difficulty. Table 2 presents the percentage of work and the results obtained by the students in the two classes.

\section{$4 \quad$ Limitations and Future Work}

The work developed so far resulted in a very useful system for teachers to monitor, in real-time, the level of attention of their students. However, limitations were also identified. In fact, if a student opens an application that is not work-related and does not interact anymore with the computer until the end of the task a level of attention of $0 \%$ is calculated. Although the student was not actually carrying out the task, she/he was also probably not even standing in front of the computer. Lack of attention and absence must needs be distinguished. Similarly, if the student opens a work-related application and does not interact with the computer after that, the attention is quantified as $100 \%$ when she/he was most likely not even at the computer. These cases must, evidently, be pointed out. In order to correctly validate the efficacy of the system it is necessary to observe the amount of time that each student devoted to work and to other activities, but also the amount of time actually spent interacting with the computer.

To address this limitation, in future work we will implement a tighter integration between the previous and the new work. Specifically, in previous work we implemented the monitoring of the interaction of the users with the keyboard and the mouse. It is thus possible to know all the actions that each user performed both with the mouse or the keyboard, and at what time. We will thus generate a new feature that will quantify the level of activity of each user throughout time. This new feature will allow a more contextualized analysis of attention, improving the performance of its classification and quantification.

In this moment, the architecture of the proposed TEL system can only analyze the student's level of attention from the percentage of time spent interacting with workrelated applications. A larger amount of data is necessary in order to analyze the profile of students and predict how each one will react in different types of lessons.

Regarding learning styles, the system only analyses the student's actions by the percentage of work-related tasks and the interaction with the mouse and the keyboard. It is possible to apply a preliminary questionnaire in order to detect the learning style of each student. When the system has enough data for each student, it will be possible to advise the teacher with the aim to improve the attention level. It will also be possible to analyze the students' profile, taking into account their individual characteristics, and to propose new strategies and actions. Given that the teacher is informed about the behavior of each student and each one's learning style, she/he will be able to maximize students' attention and, consequently, the performance of the teaching-learning process. 
With the proposed system it is possible to detect potentially negative factors dynamically and non-intrusively, making it possible to foresee negative situations, and to take actions to mitigate them. This may, in turn, minimize issues such as stress and anxiety, which can negatively influence the students' results and are closely related to the occurrence of conflicts.

The next step in this work is to integrate smartphones and tablets, taking advantage of their new features such as several incorporated sensors and high resolution cameras. This may allow a wider characterization of the student, making it possible to enhance the learning experience, through better recommendation and personalization.

\section{Acknowledgment}

This work has been supported by COMPETE: POCI-01-0145-FEDER-007043 and FCT - Fundação para a Ciência e Tecnologia within the Project Scope: UID/CEC/00319/2013.

\section{References}

1. J. M. Carroll, "Human-computer interaction: psychology as a science of design," Annual Review of Psychology, 48, 1997, 61-83. doi:10.1146/annurev.psych.48.1.61

2. R. E. Clark, "The transfer of technology between nations". In: Interaction, 1983, 1(3), 185203.

3. M. Milrad,L. H. Wong,M. Sharples,G. - J. Hwang, C. - K. Looi, H. Ogata, "Seamless Learning: An International Perspective on Next Generation Technology Enhanced Learning," Book chapter in Z. L. Berge \& L. Y. Muilenburg (eds.) Handbook of Mobile Learning, 2013, pp 95-108, New York: Routledge.

4. A. Pimenta, S. Gonçalves, D. Carneiro, F. Riverola, P. Novais, "Mental Workload Management as a Tool in e-Learning Scenarios," 2014.

5. K. Hwang, C. Yang, "Automated Inattention and Fatigue Detection System in Distance Education for Elementary School Students,” Journal of Educational Technology 12, 2009, pp. 22-35.

6. M. Mancas, "Computational Attention - Toward Attentive Computers". Press Universitaire de Louvain, 2007.

7. W. James, "Principles of Psychology, Vol.1", Dover Publications Inc., New York, 1890, pp. 255-260.

8. D. Durães, D. Carneiro, J. Bajo, P. Novais, "Using Computer Peripheral Devices to measure attentiveness," in Advances in Intelligent System and Computing, 2016, pp. 147-155. 